\title{
Determination and Monte Carlo Simulations of Neutron Flux inside Spallation Target QUINTA
}

\author{
P. Tichý ${ }^{\text {1abc }}$, J. Adam ${ }^{\text {a }}$, A. Baldin ${ }^{\text {ad }}$, P. Chudoba ${ }^{\text {be }}$, W. Furman ${ }^{\text {a }}$, S. Gustov ${ }^{\text {a }}$, \\ J. Khushvaktov ${ }^{a}$, I. Mar'in ${ }^{\text {a }}$, A. Solnyshkin ${ }^{a}$, M. Suchopár ${ }^{\text {bc }}$, J. Svoboda ${ }^{\text {af }}$, \\ S. Tyutyunnikov ${ }^{a}$, R. Vespalec ${ }^{\text {ac }}$, J. Vrzalováabc $^{\text {ab }}$ V. Wagner ${ }^{b}$, L. Závorka ${ }^{a c}$, \\ M. Zeman ${ }^{\text {af }}$
}

a Joint Institute for Nuclear Research Joliot-Curie 6, 141980 Dubna, Moscow region, Russian Federation

${ }^{b}$ Nuclear Physics Institute of the ASCR PRI

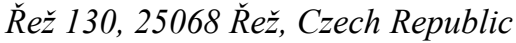

${ }^{c}$ Faculty of Nuclear Sciences and Physical Engineering, Czech Technical University in Prague Břehová 7, 11519 Prague, Czech Republic

"Institute for Advanced Studies "OMEGA"

Universitetskaya 19, 141980 Dubna, Moscow region, Russian Federation

${ }^{e}$ Faculty of Mathematics and Physics, Charles University

Ke Karlovu 3, 12116 Prague 2, Czech Republic

${ }^{f}$ Faculty of Electrical Engineering and Communication, Brno University of Technology

Technická 3058/10, 61600 Brno, Czech Republic

E-mail: tichyejinr.ru, iadamejinr.ru, an.baldinemail.ru,

chudoba@ujf.cas.cz, furmanedubna.ru, gystovenu.jinr.ru,

khushvaktovejinr.ru, mariinesunse.jinr.ru, solnejinr.ru,

suchopar@ujf.cas.cz, svobodaljinr.ru, tsi@sunse.jinr.ru,

vespalecljinr.ru, vrzalova@ujf.cas.cz, wagnereujf.cas.cz,

zavorkaejinr.ru, zemanejinr.ru

This paper deals with experiments performed with natural uranium spallation target QUINTA irradiated by $4 \mathrm{GeV}$ and $8 \mathrm{GeV}$ deuteron beam from Nuclotron accelerator and by $660 \mathrm{MeV}$ proton beam from Phasotron accelerator. Reaction rates for ${ }^{24} \mathrm{Na},{ }^{57} \mathrm{Co},{ }^{58} \mathrm{Co},{ }^{205} \mathrm{Bi}$ and ${ }^{206} \mathrm{Bi}$ production in $\mathrm{Al}, \mathrm{Co}$ and $\mathrm{Pb}$ threshold radioactivation foils were determined. The data served for calculation of the experimental neutron flux inside the QUINTA target for the Phasotron experiment. Simulations in Monte Carlo code MCNPX 2.7 were performed and compared to the experimental data. In the current state of research, the data evaluation and simulations are preliminary.

The 26th International Nuclear Physics Conference

11-16 September, 2016

Adelaide, Australia

${ }^{1}$ Speaker

(C) Copyright owned by the author(s) under the terms of the Creative Commons

Attribution-NonCommercial-NoDerivatives 4.0 International License (CC BY-NC-ND 4.0). 


\section{Introduction}

Our group in the Joint Institute for Nuclear Research (JINR) is a member of "Energy \& Transmutation of Radioactive Waste" (E\&T RAW) international collaboration. The collaboration is involved in experiments with subcritical transmutation setups. Such research is very important for accelerator driven system (ADS) and fast nuclear reactor development. The setups are composed of the lead or uranium spallation target and the uranium (natural or depleted) subcritical blanket. They are irradiated by relativistic proton or deuteron beams. In Dubna, there is a possibility to use the proton beam of energy $660 \mathrm{MeV}$ from Phasotron accelerator and also both proton and deuteron beams of energies from $1 \mathrm{GeV}$ up to $8 \mathrm{GeV}$ from Nuclotron accelerator. These beams are aimed to the subcritical targets where neutrons are created by fission and spallation reactions. These neutrons are consequently multiplied in the setup blanket. In this way, intense neutron fields are formed inside the setups.

Significant issues concerning experiments with subcritical targets are Monte Carlo simulation program benchmarks. The need for the benchmarks stems from the importance of using such codes in advanced nuclear system research where precise theoretical calculations of production and transport of neutrons are necessary.

\section{Materials and methods}

\subsection{Experimental setup}

The QUINTA setup is composed of 5 hexagonal sections with gaps for activation sample placement. Every section is $114 \mathrm{~mm}$ long and between the sections there are $17 \mathrm{~mm}$ gaps for sample placement (see figs. 1 and 2). The very front section differs from the other sections by having a free space in the middle for beam passage. The first section contains 54 rods while the other sections contain 61 rods. The uranium rods are $104 \mathrm{~mm}$ long and their diameter is $36 \mathrm{~mm}$. The weight of a single rod is $1.72 \mathrm{~kg}$ and it is encapsulated in an aluminium cover. The total weight of the natural uranium in QUINTA is $512 \mathrm{~kg}$ and the whole assembly is shielded by the lead box of $100 \mathrm{~mm}$ wall thickness.

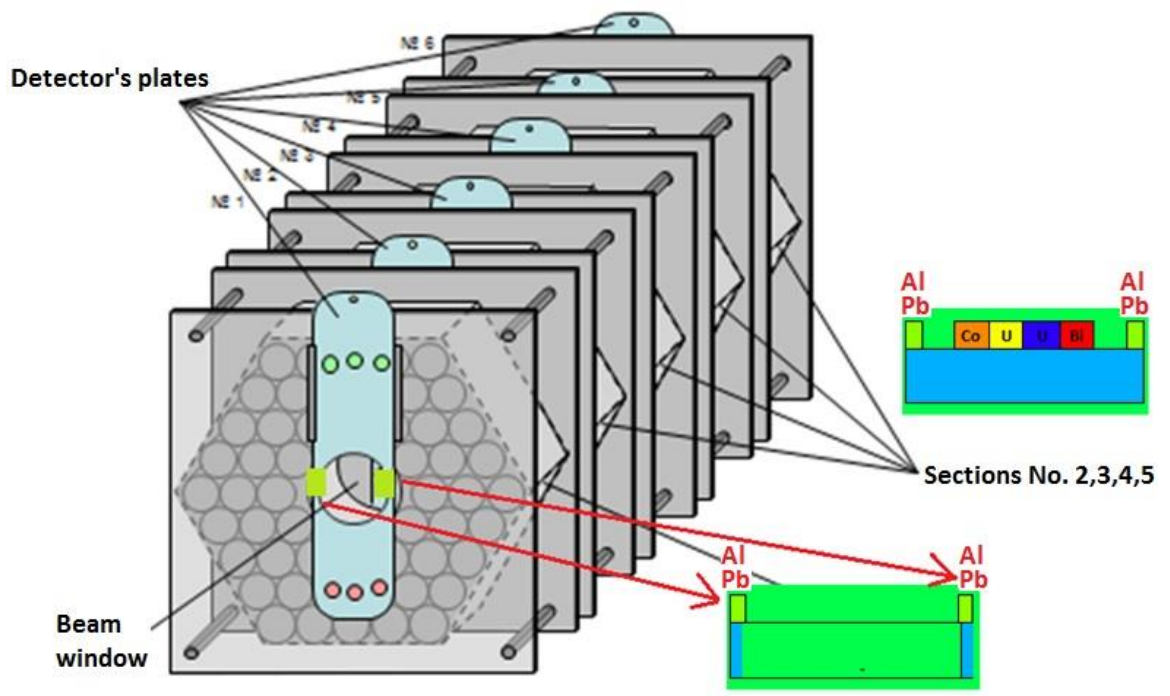

Fig. 1: QUINTA setup and placement of aluminium and lead samples from the Nuclotron experiments. The samples were placed in front of the plates, one on another, while the aluminium samples lied on the lead samples and the lead samples lied on the aluminium plates [1]. 


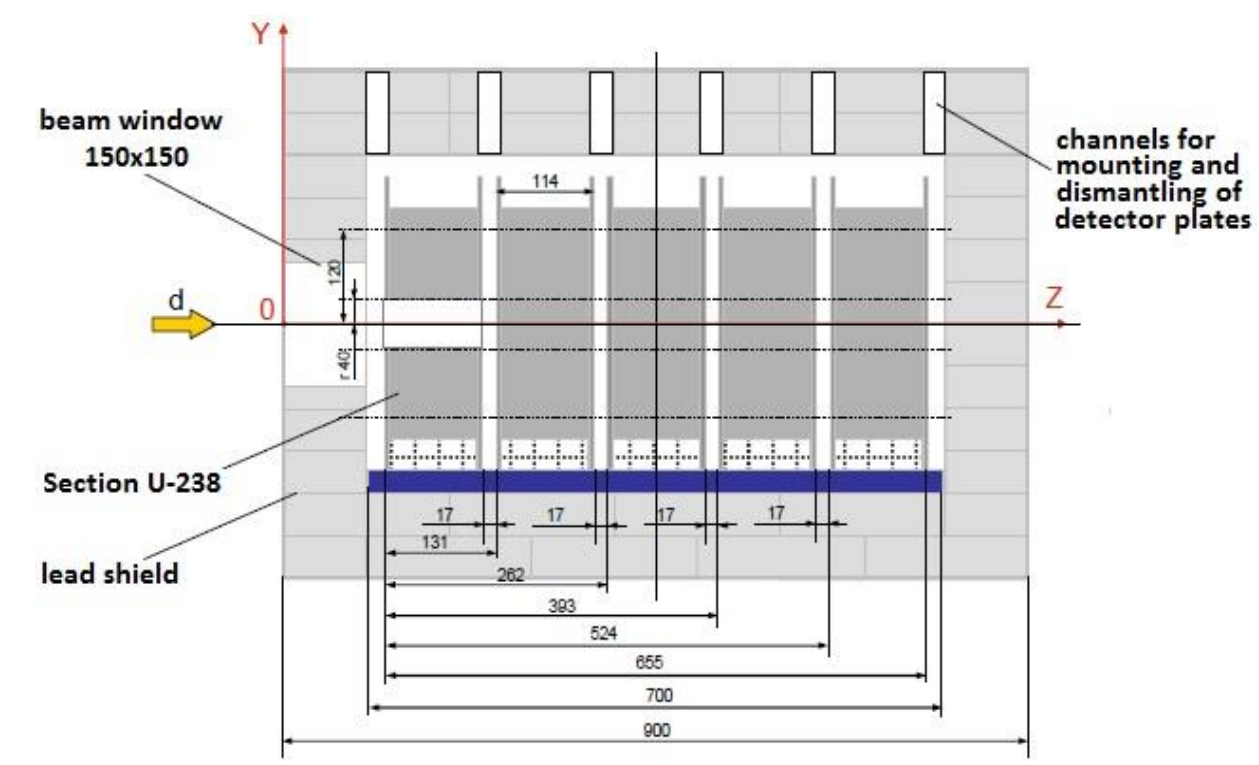

Fig. 2: Cross cut of the QUINTA setup with lead shielding (sizes in mm) [1].

\subsection{Data evaluation}

After the QUINTA assembly irradiation, the samples were taken out of QUINTA, gamma spectra were measured by HPGe detectors and consequently analysed by DEIMOS32 program developed in NPI Řež by J. Frána [2]. For determination of reaction rates $R$ (defined as total number of nuclei created in an activation sample normalised to one source particle and one atom of the sample), gamma spectrometry method was used. Our group in Dubna uses a special program package $[3,4]$.

The reaction rate $R$ can be calculated as

$$
R=N_{\text {yield }} \cdot \frac{M}{N_{A}}
$$

where $M$ is the relative atomic mass of the activation sample material, $N_{A}$ is the Avogadro constant and $N_{\text {yield }}$ is the total number of nuclei of some product created in a sample normalised to one source particle and one gram of the sample material. $N_{\text {yield }}$ is given by the equation

$$
N_{\text {yield }}=\frac{S\left(E_{\gamma}\right)}{m_{s}} \cdot \frac{1}{I_{p}} \cdot \frac{1}{I_{\gamma}} \cdot \frac{t_{\text {real }}}{t_{\text {live }}} \cdot \frac{C_{B} \cdot C_{\text {abs }}}{\varepsilon_{p}\left(E_{\gamma}\right) \cdot C_{g} \cdot C O I} \cdot \frac{\exp \left(\lambda \cdot t_{\text {delay }}\right)}{1-\exp \left(-\lambda \cdot t_{\text {real }}\right)} \cdot \frac{\lambda \cdot t_{\text {irr }}}{1-\exp \left(-\lambda \cdot t_{\text {irr }}\right)}
$$

where $m_{s}$ is the sample mass, $I_{p}$ is the integral number of the source particles, $t_{i r r}$ is the irradiation time, $t_{\text {delay }}$ is the time between end of the irradiation and start of the measurement and $\lambda$ is the decay constant.

In order to gain a number of radionuclides created in the activation samples by nuclear reactions, several corrections have to be made [5]. These corrections are: Intensity of gamma transition $I_{\gamma}$, live measurement time $\mathrm{t}_{\text {live }}\left(t_{\text {live }}=t_{\text {real }}-t_{\text {dead }}\right.$, where $\mathrm{t}_{\text {real }}$ is the real measurement time and $t_{\text {dead }}$ is the detector dead time), full-energy peak detector efficiency $\varepsilon_{p}\left(E_{\gamma}\right)$, beam instabilities during irradiation $C_{B}$, self-absorption of gamma photons in samples $C_{a b s}$, sample geometrical correction $C_{g}$ and cascade coincidence correction COI. For coincidence correction determination, there is a program TrueCoinc developed at University of Debrecen in Hungary by S. Sudár [6]. 


\subsection{Experiments on Nuclotron}

One of the experiments on Nuclotron was irradiation the QUINTA setup by deuteron beam with a total kinetic energy $4 \mathrm{GeV}$. The second experiment was irradiation the QUINTA setup by $8 \mathrm{GeV}$ deuteron beam. In both cases, activation samples of aluminium and lead were irradiated. The samples were placed on the left and right sides on each aluminium plate according to fig. 1. Particle flux is supposed to be different on the left and right side because the QUINTA was deviated from the source beam axis by 2 degrees during each experiment (for more detailed information see [7]). Some characteristics of the beam (coordinates of the beam centre where the beam impinges on the front side of the QUINTA and full energy width at half maximum (FWHM) of the 2D Gaussian beam profile) also differs during each experiment because of different accelerator settings (beam characteristics of the Nuclotron experiments described in this section and the Phasotron experiment described in section 2.4 are given in tab. 1). Dimensions of all the samples were $0.8 \times 20 \mathrm{~mm}$. Weights of the aluminium samples were about $0.20 \mathrm{~g}$ ( $4 \mathrm{GeV}$ experiment) and $0.46 \mathrm{~g} \mathrm{(8} \mathrm{GeV}$ experiment). Weights of the lead samples were about $0.50 \mathrm{~g}$ ( $4 \mathrm{GeV}$ experiment), $0.65 \mathrm{~g}$ ( $8 \mathrm{GeV}$ experiment, first 3 QUINTA sections) and $1.21 \mathrm{~g}$ ( $8 \mathrm{GeV}$ experiment, last 3 QUINTA sections).

Tab. 1: Beam characteristics of the Nuclotron experiments and the Phasotron experiment. $x$ and $y$ are coordinates of the source beam centre on the QUINTA entrance. FWHM ${ }_{x}$ and $F W H M_{y}$ are full widths at half maximums on axes $x$ and $y$ of the $2 D$ Gaussian profile on the QUINTA entrance. In case of the Nuclotron experiments, the beam parameters were measured by solid state nuclear track detectors and in case of the Phasotron experiment, the parameters were measured by ionization chambers.

\begin{tabular}{|c|c|c|c|c|}
\hline EXPERIMENT & $\mathrm{x}[\mathrm{cm}]$ & $\mathrm{y}[\mathrm{cm}]$ & $\mathrm{FWHM}_{\mathrm{x}}[\mathrm{cm}]$ & FWHM $_{\mathrm{y}}[\mathrm{cm}]$ \\
\hline 4 GeV Nuclotron & 1.40 & -1.60 & 2.70 & 1.50 \\
\hline 8 GeV Nuclotron & -0.20 & -0.70 & 2.30 & 1.10 \\
\hline 660 MeV Phasotron & 1.31 & 0.76 & 3.40 & 3.97 \\
\hline
\end{tabular}

The reason why aluminium and lead samples were chosen lies in characteristics of reactions created by interactions of protons, deuterons and neutrons with aluminium and lead. It is expected that studying of ${ }^{24} \mathrm{Na}$ nuclei production in ${ }^{27} \mathrm{Al}$ by activation techniques is able to provide us with the information about sum of neutron + proton + deuteron flux because ${ }^{24} \mathrm{Na}$ can arise from reactions with neutrons, protons and deuterons: ${ }^{27} \mathrm{Al}(\mathrm{n}, \mathrm{x}){ }^{24} \mathrm{Na},{ }^{27} \mathrm{Al}(\mathrm{p}, \mathrm{x}){ }^{24} \mathrm{Na}$ and ${ }^{27} \mathrm{Al}(\mathrm{d}, \mathrm{x}){ }^{24} \mathrm{Na}$. On the other hand, studying of ${ }^{205} \mathrm{Bi}$ and ${ }^{206} \mathrm{Bi}$ nuclei production in ${ }^{\text {nat }} \mathrm{Pb}$ gives the information about sum of proton + deuteron flux because ${ }^{205} \mathrm{Bi}$ and ${ }^{206} \mathrm{Bi}$ can arise only from reactions with protons and deuterons: ${ }^{\text {nat }} \mathrm{Pb}(\mathrm{p}, \mathrm{x})^{206(205)} \mathrm{Bi}$ and ${ }^{\text {nat }} \mathrm{Pb}(\mathrm{d}, \mathrm{x})^{206(205)} \mathrm{Bi}$. It is physically impossible the ${ }^{205} \mathrm{Bi}$ or ${ }^{206} \mathrm{Bi}$ to be created by reactions with neutrons because of lead $(\mathrm{Z}=82)$ and bismuth $(\mathrm{Z}=83)$ proton numbers.

After the reaction rates of production of ${ }^{24} \mathrm{Na},{ }^{205} \mathrm{Bi}$ and ${ }^{206} \mathrm{Bi}$ were analysed, theoretical calculations were also made. Simulations of neutron flux in aluminium samples and simulations of proton and deuteron flux in lead samples were performed in Monte Carlo simulation code MCNPX version 2.7 [8]. Neutron data library used for the simulations was ENDF70 [9] and physics models were INCL4.2 [10] + ABLA-KHSv3p [11,12]. Cross sections of above mentioned reactions were calculated by deterministic code TALYS version 1.6 [13]. Reaction rates of production of ${ }^{24} \mathrm{Na},{ }^{205} \mathrm{Bi}$ and ${ }^{206} \mathrm{Bi}$ were then gained as convolutions of simulated flux and calculated cross sections. 
For simulations (both for Nuclotron and Phasotron experiments), experimentally evaluated beam centre positions as well as deuteron (proton) intensity Gaussian distribution parameters of the beam were used in accordance with tab. 1. Also the 2 degree QUINTA deviation mentioned above was taken into consideration.

\subsection{Experiment on Phasotron}

In the experiment on Phasotron, the QUINTA setup was irradiated by the proton beam of energy $660 \mathrm{MeV}$. Samples of cobalt and lead were irradiated. Positions of the samples on the left and right side, determination and calculation of theoretical values were done in the same way as in the case of Nuclotron experiments as described in the previous section, but instead of aluminium samples cobalt samples were used. Production of ${ }^{57} \mathrm{Co}$ and ${ }^{58} \mathrm{Co}$ was studied. The ${ }^{57} \mathrm{Co}$ and ${ }^{58} \mathrm{Co}$ arise from reactions with neutrons: ${ }^{59} \mathrm{Co}(\mathrm{n}, 2 \mathrm{n}){ }^{58} \mathrm{Co},{ }^{59} \mathrm{Co}(\mathrm{n}, 3 \mathrm{n}){ }^{57} \mathrm{Co}$ and with protons: ${ }^{59} \mathrm{Co}(\mathrm{p}, \mathrm{x}){ }^{58(57)} \mathrm{Co}$. Dimensions of the cobalt samples were $8 \times 25 \mathrm{~mm}$ and of the lead samples $8 \times 20 \mathrm{~mm}$. Weights of the cobalt samples were about $1.95 \mathrm{~g}$ and of the lead samples about $0.51 \mathrm{~g}$.

The approximate experimental neutron flux $\phi_{n}$ in the energy interval from $10.63 \mathrm{MeV}$ (threshold of ${ }^{59} \mathrm{Co}(\mathrm{n}, 2 \mathrm{n}){ }^{58} \mathrm{Co}$ reaction), alternatively from $19.35 \mathrm{MeV}$ (threshold of ${ }^{59} \mathrm{Co}(\mathrm{n}, 3 \mathrm{n})^{57} \mathrm{Co}$ reaction), was evaluated (in the positions where the samples were placed) by the following method [14].

Proton flux $\phi_{p}$ is given by

$$
\phi_{p}=\frac{R\left({ }^{206} B i\right)}{\sigma_{p}^{e f}\left({ }^{206} B i\right)}
$$

where $R\left({ }^{206} \mathrm{Bi}\right)$ is the experimental reaction rate for ${ }^{206} \mathrm{Bi}$ production and $\sigma_{p}^{e f}\left({ }^{206} \mathrm{Bi}\right)$ is the effective cross section of ${ }^{\text {nat }} \mathrm{Pb}(\mathrm{p}, \mathrm{x})^{206} \mathrm{Bi}$ reaction calculated as

$$
\sigma_{p}^{e f}\left({ }^{206} B i\right)=\frac{\Sigma \sigma_{i}^{p} \phi_{i}^{p}}{\Sigma \phi_{i}^{p}}
$$

where $\sigma_{i}^{p}$ is the partial cross section of the ${ }^{\text {nat }} \mathrm{Pb}(\mathrm{p}, \mathrm{x}){ }^{206} \mathrm{Bi}$ reaction for a given energy interval $i$ calculated by TALYS 1.6 and $\phi_{i}^{p}$ is the flux of protons for interval $i$ simulated by MCNPX 2.7.

Because the cobalt and lead samples were situated in the same positions inside QUINTA and thus the proton spectra can be considered the same for cobalt and lead samples, one can write

$$
R_{p}\left({ }^{58} \mathrm{Co}\right)=\sigma_{p}^{e f}\left({ }^{58} \mathrm{Co}\right) \cdot \phi_{p}
$$

where $R_{p}\left({ }^{58} \mathrm{Co}\right)$ is the reaction rate and $\sigma_{p}^{e f}\left({ }^{58} \mathrm{Co}\right)$ is the effective cross section of ${ }^{59} \mathrm{Co}(\mathrm{p}, 2 \mathrm{n}){ }^{58} \mathrm{Co}$ reaction. Reaction rate $R_{n}\left({ }^{58} \mathrm{Co}\right)$ for ${ }^{59} \mathrm{Co}(\mathrm{n}, 2 \mathrm{n}){ }^{58} \mathrm{Co}$ reaction is given by

$$
R_{n}\left({ }^{58} \mathrm{Co}\right)=R\left({ }^{58} \mathrm{Co}\right)-R_{p}\left({ }^{58} \mathrm{Co}\right)
$$

where $R\left({ }^{58} \mathrm{Co}\right)$ is the experimental reaction rate for ${ }^{58} \mathrm{Co}$ production. Then the neutron flux $\phi_{n}$ can be calculated according to the equation

$$
\phi_{n}=\frac{R_{n}\left({ }^{58} \mathrm{Co}\right)}{\sigma_{n}^{e f}\left({ }^{58} \mathrm{Co}\right)}
$$

where $\sigma_{n}^{e f}\left({ }^{58} \mathrm{Co}\right)$ is the effective cross section of ${ }^{59} \mathrm{Co}(\mathrm{n}, \mathrm{x}){ }^{58} \mathrm{Co}$ reaction.

The method described above was not applied for the Nuclotron experiments because the deuteron contributions to reaction rates of bismuth production were high (usually less than $5 \%$ ) and neclecting the deuteron contribution would cause a significant error. 


\section{Results and discussion}

In this section, some representative results are shown. Ratios of the left and right side results of the reaction rates of the Nuclotron experiments are presented in fig. 3, and of the Phasotron experiment in fig. 4. Ratios of experimental to simulated reaction rates of ${ }^{24} \mathrm{Na}$ production of the $4 \mathrm{GeV}$ Nuclotron experiment are shown in fig. 5. Experimental and simulated neutron flux for the left side of the Phasotron experiment is given in fig. 6. Statistic uncertainties of the experimental results are shown in the graphs.

From the graph 3 of the left and right side result ratios can be noticed that the ratios have an increasing tendency towards greater longitudinal distance. The highest ratio values can be found for ${ }^{205} \mathrm{Bi}$ and ${ }^{206} \mathrm{Bi}$ production from the $4 \mathrm{GeV}$ Nuclotron experiment. Ratio value of about 4 was reached at longitudinal distance $67.15 \mathrm{~cm}$ for ${ }^{206} \mathrm{Bi}$ production. This is caused by the effect of the 2 degree QUINTA deviation from the source beam axis and by the shift of the source beam centre dependable on accelerator settings [7].

Graph 4 of the left and right side ratios shows maximum at longitudinal distance $40.95 \mathrm{~cm}$ opposite to the graph 4 of Nuclotron experiments where just increasing tendency is shown and thus no maximum can be found.

From graph 5 one can notice that greater values of experimental reaction rates are shown on the left side.

Discrepancies between experimental and simulated neutron fluxes of the Phasotron experiment are evident from fig. 6 .

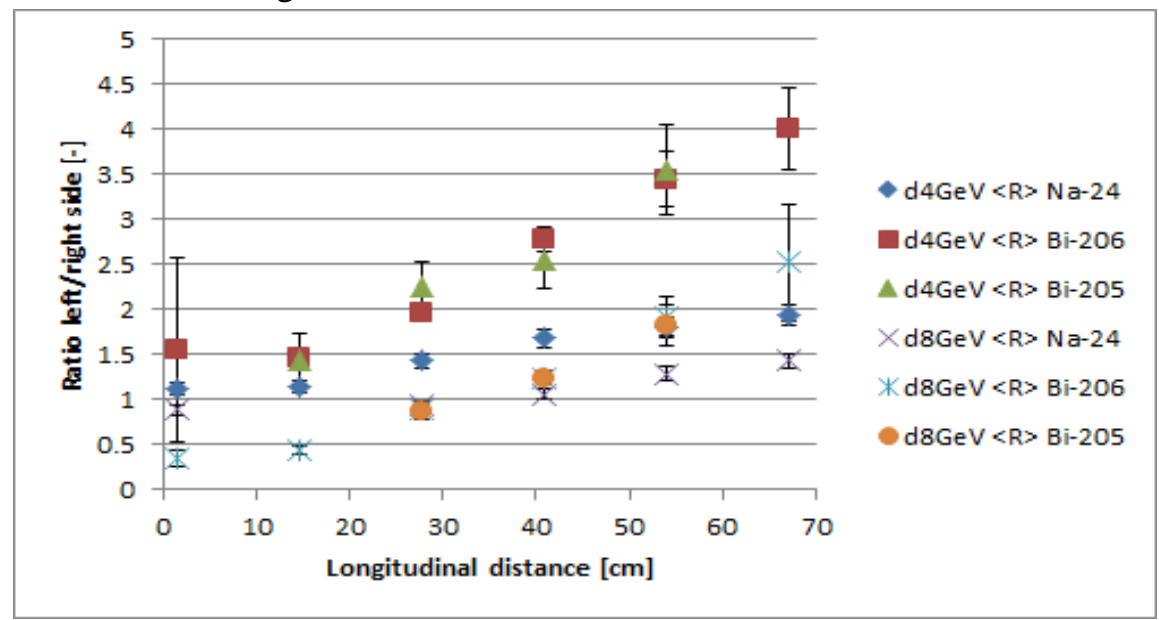

Fig. 3: Left/right side experimental reaction rate ratios for the Nuclotron experiments.

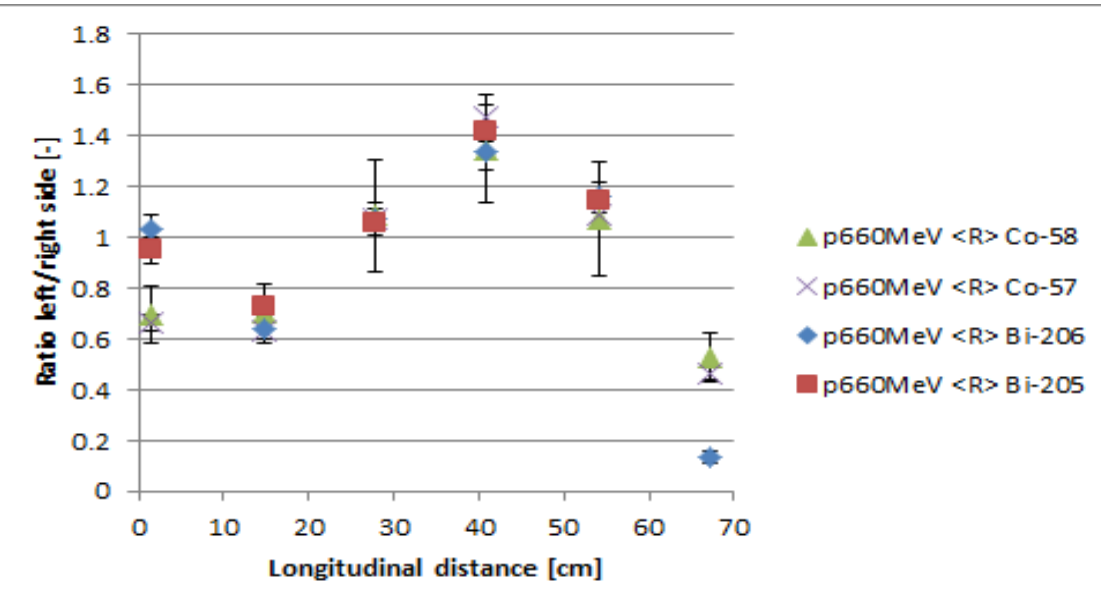

Fig. 4: Left/right side experimental reaction rate ratios for the Phasotron experiment. 


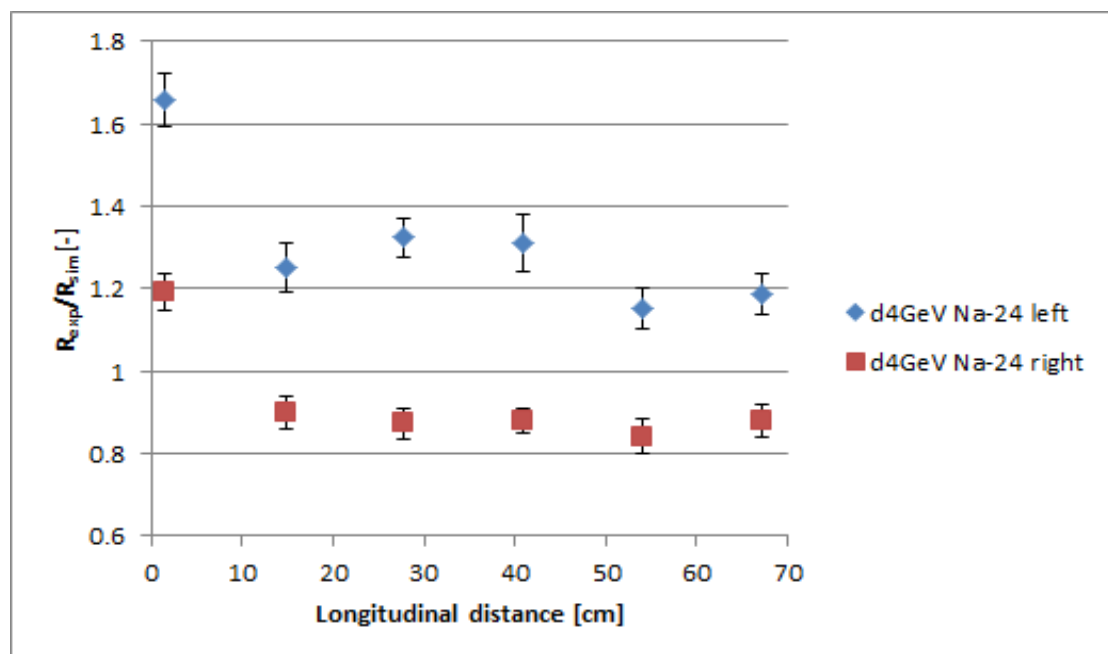

Fig. 5: Ratios of experimental and simulated reaction rates of production of ${ }^{24} \mathrm{Na}$ from the $4 \mathrm{GeV}$ Nuclotron experiment. Mean values for the left and right side were

$<R_{\text {exp }} / R_{\text {sim }}>($ left $)=1.31,<R_{\text {exp }} / R_{\text {sim }}>($ right $)=0.93$.

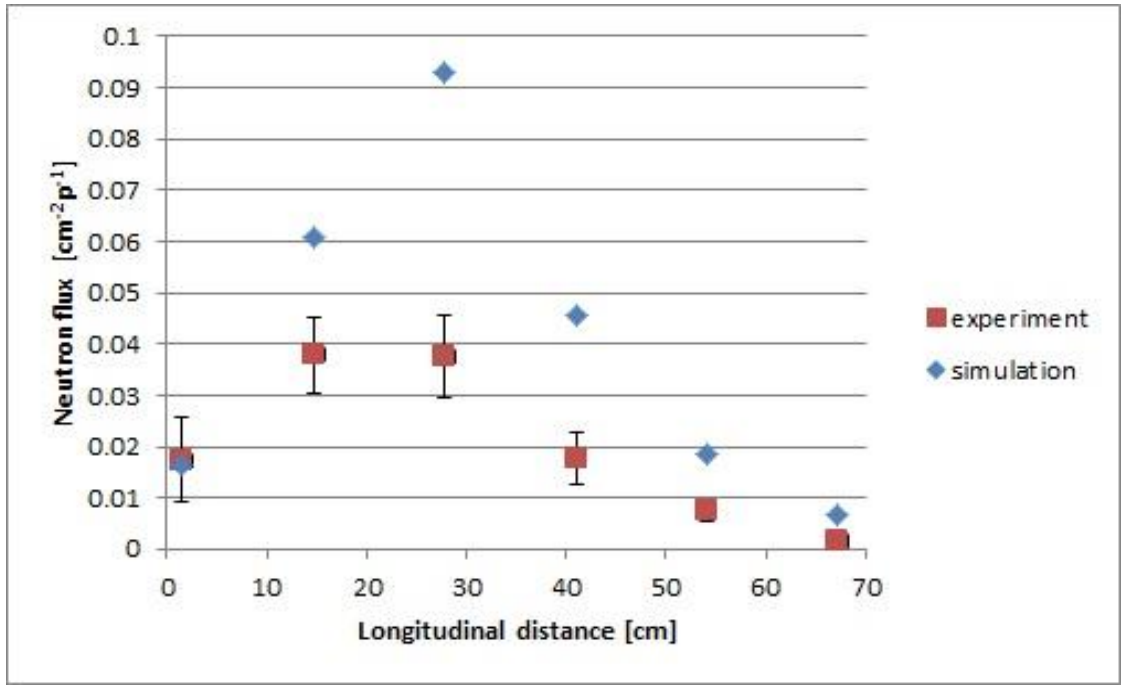

Fig. 6: Experimental and simulated neutron flux on the left side for the Phasotron experiment.

\section{Conclusion}

Reaction rates of ${ }^{24} \mathrm{Na},{ }^{57} \mathrm{Co},{ }^{58} \mathrm{Co},{ }^{205} \mathrm{Bi}$ and ${ }^{206} \mathrm{Bi}$ from the QUINTA experiments on Nuclotron and Phasotron were determined and also experimental neutron flux inside the QUINTA target for the Phasotron experiment was assessed. Simulations in Monte Carlo code MCNPX 2.7 were performed and compared to the experimental data.

For the Phasotron experiment, experimentally was evaluated that the ratio of proton and neutron flux at longitudinal distance $40.95 \mathrm{~cm}$ for the right side was 0.124 . According to simulations, the ratio was $1.84 \mathrm{E}-2$. For the $4 \mathrm{GeV}$ Nuclotron experiment, the ratio of simulated deuteron and neutron flux in the same position was $1.48 \mathrm{E}-4$, for the $8 \mathrm{GeV}$ Nuclotron experiment the ratio was 3.12E-5.

Considerable amount of protons was present in various positions of the QUINTA setup, even in the most remote position (last aluminium plate). The reason is that there is much free space between the QUINTA natural uranium rods. Even the 2 degree setup deviation does not guarantee that all passing protons will be absorbed. 
There exist significant differences between experimental and simulated results (figs. 5 and 6). This seems to be caused by imperfect description of beam centre position and beam direction. Precise analysis of influence of beam parameter determination on the results needs to be made before we will be able to assess the precision of the MCNPX code as well as the precision of the nuclear data libraries (particularly neutron, proton and deuteron cross sections) properly. Therefore the results presented in this paper are still preliminary.

\section{References}

[1] W. Furman et al, Recent results of the study of ADS with $500 \mathrm{~kg}$ natural uranium target assembly QUINTA irradiated by deuterons with energies from 1 to $8 \mathrm{GeV}$ at JINR NUCLOTRON, Proceedings of Science, Baldin ISHEPP XXI, Dubna, Russia, 2012. No. 086.

[2] J. Frána, Program DEIMOS32 for gamma-ray spectra evaluation, Journal of Radioanalytical and Nuclear Chemistry, Akadémia Kiadó, 2003. Vol. 257, pp. 583-587.

[3] J. Adam, Program Package and Supplements to Activation Analysis for Calculations of Nuclear Reaction Cross-Sections, JINR Preprint P10-10-2000-28, 2000.

[4] R. Vlespalec et al, YASNAPP-2 Program Package for Data Analysis 1.4 (YAPP 1.4): User's Manual, unpublished, 2016.

[5] J. Vrzalová, Experimental determination of neutron reaction cross sections relevant for acceleratordriven transmutation systems (in Czech), Ph.D. thesis, CTU, Prague, 2015.

[6] S. Sudár, USER GUIDE TrueCoinc: A program for calculation of true coincidence correction for gamma rays, online, accessed 21-October-2016. [http://kisfiz.phys.klte.hu/kisfiz/sudar/TrueCoinc/UserGuide.pdf]

[7] L. Závorka, Transmutation of Actinides Using Spallation Reactions, Ph.D. thesis, CTU, Prague, 2015.

[8] D. B. Pelowitz, MCNPX Users Manual Version 2.7.0, LANL, 2011.

[9] H. R. Trellue et al, ENDF70: A continuous-energy MCNP neutron data library based on ENDF/BVII.0, Nuclear Technology, 2009. pp. 832-836.

[10] A. Boudard and J. Cugnon, INCL4-The Liege INC model for high-energy hadron-nucleus reactions, Joint ICTP-IAEA Workshop on Model Codes for Spallation Reactions, Trieste, Italy, 2008.

[11] J. J. Gaimard and K. H. Schmidt, A reexamination of the abrasion-ablation model for the description of the nuclear fragmentation reaction, Nuclear Physics A, Elsevier, 1991. pp. 709-745.

[12] A. R. Junghans et al, Projectile-fragment yields as a probe for the collective enhancement in the nuclear level density, Nuclear Physics A, Elsevier, 1998. pp. 635-655.

[13] A. Koning et al, TALYS-1.6: A Nuclear Reaction Program, User Manual, NRG, 2003.

[14] J. S. Wan et al, Transmutation of radioactive waste by means of relativistic heavy ions, Kerntechnik, 1998. pp. 167-177.

[15] P. Tichý and M. Suchopár, Future usage of quasi-infinite depleted uranium target (BURAN) for benchmark studies, Proceedings of Science, Baldin ISHEPP XXII, Dubna, Russia, 2014. No. 065. 